\title{
A NEW COMPARISON THEOREM FOR SCALAR RICCATI EQUATIONS ${ }^{1}$
}

\author{
BY R. A. STAFFORD AND J. W. HEIDEL
}

Communicated by Fred Brauer, November 20, 1973

Consider the Riccati equation

$$
\dot{r}+r^{2}+q_{1}(t)=0
$$

where $q_{1}(t)$ is continuous on some interval $[a, b)$ with $0 \leqq a<b \leqq \infty$. The problem is to find conditions on $q_{1}(t)$ under which (1) will or will not have a solution existing on $[a, b)$. This is equivalent to the disconjugacy problem for $\ddot{y}+q_{1}(t) y=0$ via $r(t)=\dot{y}(t) / y(t)$. Here we announce a comparison theorem which relates the existence of a solution of (1) to the existence of a solution of another equation,

$$
\dot{s}+s^{2}+q_{2}(t)=0 .
$$

Specific criteria are then obtained by choosing $q_{1}$ or $q_{2}$ so that a solution of (1) or (2) can be exhibited.

The basic idea is exposed by proving a simple form of the theorem. Examples and the relation to other comparison theorems will then be discussed. A generalization and further examples are considered in [5]. Extension to matrix Riccati equations is given in [3].

THEOREM 1. Suppose that $q_{1}$ and $q_{2}$ are continuous and nonnegative on $[a, b)$ where $0<a<b \leqq \infty$ and that $\int_{a}^{t} \tau^{2} q_{2}(\tau) d \tau \leqq \int_{a}^{t} \tau^{2} q_{1}(\tau) d \tau, t \geqq a$. If (1) has a solution $r(t)$ on $[a, b)$ and $\operatorname{ar}(a)<1$, then (2) has a solution on $[a, b)$.

Proof. The substitutions $u(t)=\operatorname{tr}(t)$ and $v(t)=t s(t)$ transform (1) and (2) into

$$
\begin{aligned}
& t \dot{u}=u-u^{2}-t^{2} q_{1}, \\
& t \dot{v}=v-v^{2}-t^{2} q_{2}
\end{aligned}
$$

respectively. We have $u(a)=\operatorname{ar}(a)<1$. Choose a solution $v(t)$ of (4) such that $u(a)<v(a)<1$. It will be shown that $v(t)$ exists on $[a, b)$.

AMS (MOS) subject classifications (1970). Primary 34A30, 34C05.

Key words and phrases. Riccati equation, comparison theorem, disconjugacy.

${ }^{1}$ Supported in part by NSF GP 39113 and by 1410522350 R95. 
First of all it is asserted that $v(t) \leqq 1$ for $t \geqq a$ (as long as $v$ exists). This follows immediately from (4) by noting that $\dot{v} \leqq 0$ if $v \geqq 1$.

It is now asserted that $v(t)>u(t)$ for $t \geqq a$ (as long as $v$ exists). To see this integrate by parts the left-hand sides from $a$ to $t$ and subtract (4) from (3) to obtain

$$
\begin{aligned}
v(t)-u(t)= & (a / t)(v(a)-u(a)) \\
& +\frac{1}{t} \int_{a}^{t}\left\{\left(2 v(\tau)-v^{2}(\tau)\right)-\left(2 u(\tau)-u^{2}(\tau)\right)\right\} d \tau \\
& +\frac{1}{t}\left\{\int_{a}^{t} \tau^{2} q_{1}(\tau) d \tau-\int_{a}^{t} \tau^{2} q_{2}(\tau) d \tau\right\} .
\end{aligned}
$$

Suppose that $v\left(t_{1}\right)=u\left(t_{1}\right)$ for some $t_{1}>a$ and that $u(t)<v(t)$ for $a \leqq t<t_{1}$. Replacing $t$ by $t_{1}$ in (5) the first term on the right-hand side is positive and the last term is nonnegative. To examine the middle term consider the function $h(u)=2 u-u^{2}$. Its maximum occurs at $u=1$ and $h^{\prime}(u)>0$ for $u<1$. Since $u(\tau) \leqq v(\tau) \leqq 1$ for $a \leqq \tau \leqq t_{1}$, it follows that $2 u(\tau)-u^{2}(\tau)<2 v(\tau)-$ $v^{2}(\tau)$ for $a \leqq \tau \leqq t_{1}$. Thus the second term on the right-hand side is also nonnegative which contradicts $v\left(t_{1}\right)-u\left(t_{1}\right)=0$.

It has been shown that $u(t)<v(t) \leqq 1$ as far as $v$ exists. Thus $v$ exists on $[a, b)$.

EXAMPLE 1. Setting $q_{1}(t)=1 /\left(4 t^{2}\right)$ we have $r(t)=1 /(2 t)$ and $\operatorname{tr}(t)=\frac{1}{2}$. The integral criterion becomes

$$
\frac{1}{t-a} \int_{a}^{t} \tau^{2} q_{2}(\tau) d \tau \leqq \frac{1}{4} .
$$

$q_{2}(t)=1 /\left(4 t^{2}\right)[1-\sin (t-a)]$ provides a simple explicit example which satisfies this integral condition for all $t \geqq a$.

EXAMPLE 2. More generally it can be shown that $\ddot{y}+\left(k / t^{2}\right)(1+f(t)) y=0$ where $f(t) \geqq-1$, continuous, periodic of period $\omega$, and $\int_{a}^{\omega+a} f(t) d t=0$, is disconjugate on $[a, \infty)$ if $k \leqq \frac{1}{4}$ and oscillatory on $[a, \infty)$ if $k>\frac{1}{4}$.

It is interesting to compare the above theorem with other comparison theorems.

STURM'S COMPARISON THEOREM [6]. If $q_{2}(t) \leqq q_{1}(t)$ on $[a, b)$ and (1) has a solution on $[a, b)$ then (2) has a solution on $[a, b)$.

HILLE'S COMPARISON THEOREM [2] (As generalized by Hartman [1, p. 369]). If $b=\infty$,

$$
\left|\int_{t}^{\infty} q_{2}(\tau) d \tau\right| \leqq \int_{t}^{\infty} q_{1}(\tau) d \tau, \quad t \geqq a,
$$

and (1) has a solution on $[a, b)$ then (2) has a solution on $[a, b)$. 
LEVIN'S COMPARISON THEOREM [4]. If

$$
\left|\int_{a}^{t} q_{2}(\tau) d \tau-s_{0}\right| \leqq \int_{a}^{t} q_{1}(\tau) d \tau-r(a)
$$

where $r(t)$ is a solution of (1) on $[a, b)$, then the solution $s(t)$ of (2) with $s(a)=s_{0}$ exists on $[a, b)$.

We note that none of these three theorems can handle the $q_{2}$ in Example 1. This is immediately clear for Sturm's theorem and Hille's theorem. In the case of Levin's theorem we see that $r(a) \leqq 0$ so that $r(t)$ has finite escape time and hence the theorem does not give a result on $[a, \infty)$.

Wong [7] has a comparison theorem which, like Hille's theorem, involves integrals on $[t, \infty)$ and does not apply to the above example. The authors are not aware of any other comparison theorems for disconjugacy.

We now state a result which generalizes Theorem 1 in two directions.

THEOREM 2 [5]. Suppose there exists a continuous function $\mu$ on $[a, b)$ such that $\mu(t)>0$ and $\int_{a}^{t} \mu^{2}(\tau) q_{2}(\tau) d \tau \leqq \int_{a}^{t} \mu^{2}(\tau) q_{1}(\tau) d \tau, t \geqq a$.

If $(1)$ has a solution $r(t)$ on $[a, b), \mu(t) r(t) \leqq \dot{\mu}(t)$ and

$$
2 \ddot{\mu}+2(\dot{\mu}-\mu r)^{2} / \mu+\mu q_{1}+\mu q_{2} \geqq 0, \quad t \geqq a,
$$

then (2) has a solution on $[a, b)$.

EXAMPLE 3. Let $\mu(t)=t$ and $q_{1}(t)=1 /(4 t)^{2}$. Then (6) becomes $-3 / 4 t^{2} \leqq$ $q_{2}(t)$ which relaxes the nonnegativity condition of Example 1 .

EXAMPLE 4. If $q_{2}(t) \geqq 0$ and continuous on $[a, b), \gamma \geqq \frac{3}{4}$ and

$$
\int_{a}^{t} \tau^{2 \gamma} q_{2}(\tau) d \tau \leqq \frac{1}{4(2 \gamma-1)}\left(t^{2 \gamma-1}-a^{2 \gamma-1}\right), \quad t \geqq a,
$$

then (2) has a solution on $[a, b)$.

In conclusion it is interesting to note that the differential equation (3), used in the proof of Theorem 1, is the differentiated form of the integral equation used by Hille to derive his comparison theorem. Another curious relation in this regard is the identity

$$
\frac{1}{t} \int_{0}^{t} \tau^{2} q(\tau) d \tau+t \int_{t}^{\infty} q(\tau) d \tau=\frac{2}{t} \int_{0}^{t} \tau \int_{\tau}^{\infty} q(\xi) d \xi d \tau
$$

which holds for every continuous function $q(t)$ on $[0, \infty)$ such that $\int_{0}^{\infty} q(\tau) d \tau<\infty$. 


\section{REFERENCES}

1. Philip Hartman, Ordinary differential equations, Wiley, New York, 1964. MR 30 \#1270.

2. Einar Hille, Non-oscillation theorems, Trans. Amer. Math. Soc. 64 (1948), 234 252. MR 10, 376.

3. R. A. Jones, Existence theorems for matrix Riccati equations, Ph.D. Dissertation, University of Tennessee, 1973.

4. A. Ju. Levin, A comparison principle for second-order differential equations, Dokl. Akad. Nauk SSSR 135 (1960), 783-786=Soviet Math. Dokl. 1 (1960), 1313-1316. MR 23 \#A1875.

5. R. A. Stafford, Existence criteria for scalar Riccati equations, Ph.D. Dissertation, University of Tennessee, 1974.

6. C. Sturm, Sur les équations differentielles lineares du second ordre, J. Math. Pures Appl. 1 (1836), 106-186.

7. James S. W. Wong, Oscillation and nonoscillation of solutions of second order linear differential equations with integrable coefficients, Trans. Amer. Math. Soc. 144 (1969), 197-215. MR 40 \#4536.

Department of Mathematics, University of Tennessee, Knoxville, Tennessee 37916 